\title{
Real Time 3D Face Alignment with Random Forests-based Active Appearance Models
}

\author{
Gabriele Fanelli, Matthias Dantone, Luc Van Gool \\ Computer Vision Laboratory, ETH Zurich, \\ Sternwartstrasse 7, 8092 Zurich, Switzerland \\ $\{$ fanelli/dantone/vangool\}@vision.ee.ethz.ch
}

\begin{abstract}
Many desirable applications dealing with automatic face analysis rely on robust facial feature localization. While extensive research has been carried out on standard 2D imagery, recent technological advances made the acquisition of 3D data both accurate and affordable, opening new ways to more accurate and robust algorithms. We present a modelbased approach to real time face alignment, fitting a 3D model to depth and intensity images of unseen expressive faces. We use random regression forests to drive the fitting in an Active Appearance Model framework. We thoroughly evaluated the proposed approach on publicly available datasets and show how adding the depth channel boosts the robustness and accuracy of the algorithm.
\end{abstract}

\section{INTRODUCTION}

Future human-computer interfaces will likely use vision to understand the user's movements and commands. In gaming, systems like Microsoft Kinect can already track body movements; however, facial movements also carry a great deal of important information in human-human communication and future smart interfaces should be able to interpret nods, facial expressions, or recognize the identity of the user. A necessary step for virtually all applications based on automatic face analysis is the localization of key features like eyes, nose, mouth, eyebrows, and face contours.

Most of the works in the literature so far have focused on standard images and videos, which present still unsolved challenges like changes in illumination conditions or the lack of texture in some areas of the face.

Today, both accurate [1] and affordable depth-sensing devices (like MS Kinect) are available, providing a valuable new cue for overcoming the above problems inherent to methods solely based on 2D imagery. However, up to now, 3D data has mainly been leveraged for tracking [2], [3], [4], leaving open issues such as drift and initialization. To improve robustness, tracking-by-detection methods, working on each frame independently, are desirable, if only for reinitializing tracking when lost.

We present a system for real time, frame-based alignment of a generic face model to a scan of an unseen person. As first proposed in [5], we couple Active Appearance Models (AAMs) [6] with Random Regression Forests (RRFs) [7] and propose to leverage the depth channel in addition to standard intensity images to achieve a fast and robust algorithm.

The authors acknowledge the EU projects RADHAR (FP7-ICT-248873) and TANGO (FP7-ICT-249858), and the SNF project Vision-supported Speech-based Human Machine Interaction (200021-130224).

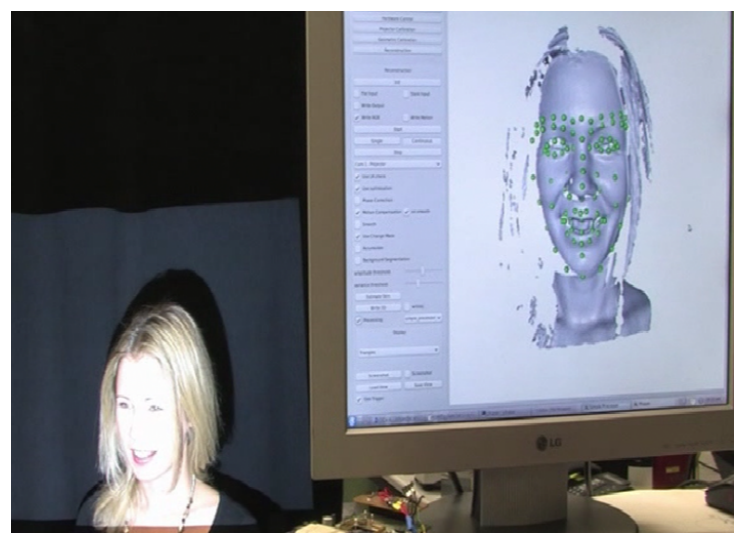

Fig. 1. Our system working in real time on the output of a 3D scanner.

\section{RELATED WORKS}

Facial feature detection from 2D images and video is an active area of research, usually aimed at preprocessing the data in order to achieve better recognition. Most methods can be classified into two main categories, depending on whether they use global or local features.

Holistic methods like Active Appearance Models (AAMs) [6] fit a generative model to a test sample using the texture of the whole face. AAMs have been extensively used in the community and represent one of the most established "interpretation through synthesis" approaches, especially for face analysis. They are fit to a new image by minimizing a measure of the error between the reconstructed texture model and the input image under the current instance of the shape model. The optimization happens within a standard patch, or shape-free image, defined by the average shape. Many modifications to the original algorithm were introduced over the years, for example proposing different fitting algorithms [8], handling multiple views [9], or extending the shape model to 3D [10]. The main shortcomings of AAM-related approaches, especially when applied to standard intensity images, are a consequence of the complexity of the appearance modeling. In general, they are sensitive to lighting condition changes and perform poorly on identities which differ substantially from the ones seen at training time [11].

Recently, methods based on independent feature detectors [12], [13], [14] have become popular. These detectors are 
discriminative models of the facial appearance within small patches centered around the landmarks. These suffer from ambiguity as the support region is too small to cope with the large appearance variations and global models need to be used to constrain the face configuration to something plausible. Such models can be explicit, as in Active Shape Models (ASMs) [15] or Constrained Local Models (CLMs) [16], [17], [18], or implicitly learned from the data [19].

Given the recent developments of depth-sensing devices, several approaches exist which leverage the depth cue for facial features localization. In [20], the 3D Statistical Facial feature Model (SFAM) is proposed, which models both the global variations in the morphology of the face and the local structures around the landmarks. Only neutral faces are taken into account and 15 minutes are needed to localize 15 landmarks. Another slow approach is the one of [21], where fitting the proposed PCA shape model (containing only the upper facial features), takes on average 2 minutes. Many works use surface curvatures, e.g., the authors of [22] use them to localize the inner corners of the eyes. Such an approach is sensitive to missing data, particularly the inner eye corners, easily occluded by shadows. The authors of [23] detect a set of 14 fiducials using a dictionary of local shapes, characterized by a set of different descriptors, with a runtime of several seconds per scan. Using personalized models built with some manual intervention, the system of [2] tracks faces in detail and in real time from high quality scans; the extension to use RGB-depth streams provided by commercial sensors like Kinect [3] also needs person-specific training. The work of [18] recently extended the CLM algorithm to using both RGB and depth channels coming from a Kinect.

Random Forests (RFs) [7] enjoy a great success in many fields within the computer vision community: object detection and action recognition [24], human pose estimation [25], [26], [27], real time head pose estimation [28], and facial expression recognition [29]. In our recent work, random forests have been successfully employed also for facial feature detection, both from standard images [19] and from 3D scans [28]: Image patches independently vote for the location of a number of facial landmarks, whose final position is found through mean-shift.

Combining AAMs and RFs was initially proposed by [5], applied to the alignment of 2D face models to grey scale images. The paper builds on an idea first described in [30], where the so-called Sequential AAMs are comprised of different regressors, trained in sequence, with increasing complexity. The authors of [5] compared RFs and Boosting as general-purpose regressors for learning a mapping between the sampled image intensity under the current model's parameters and additive updates to the shape parameters. Two ways are presented for training such combinations of regressors: Coupled Sequential AAMs and Independent Sequential $A A M s$. The former approach trains each regressor using the output of the previous one in the sequence, which suffered from over fitting. Independent Sequential AAMs, instead, train their regressors on databases obtained by deforming different sets of parameters by hand-picked amounts.
In this paper, we extend the idea of Independent Sequential AAMs using Random Forests to the task of robust, frameindependent, real time facial features localization when both intensity and depth data is available. We learn a mapping between the input data (depth and grey scale images) and continuous updates to the PCA shape model parameters. Our system works in real time without the aid of parallel processing. The only requirement is a rough initialization, for which, when depth data is available, different methods exist like [28] and [31]. In our experiments, we show that the proposed method achieves low errors and can handle unseen faces and large facial deformations due to expression.

\section{METHOD}

Active Appearance Models are linear models of the shape and the texture of an object (e.g., the face), constructed from aligned annotated images through Principal Component Analysis (PCA).

Defining a shape as the vector containing the $k$ landmarks' coordinates $\mathbf{s}=\left[x_{0}, y_{0}, z_{0}, x_{1}, y_{1}, z_{1}, \ldots x_{k}, y_{k}, z_{k}\right]$, the linear shape model is then:

$$
\mathbf{s}=\mathbf{s}_{0}+\sum_{i=1}^{n} b_{i} \mathbf{s}_{i}
$$

where $\mathbf{s}_{0}$ is the average shape and $\mathbf{s}_{i}$ are the shape eigenvectors, with $n<3 k$. The shape parameters $b_{i} \in \mathbf{b}$ control the deformations of the model according to the variation seen at train time, and are usually constrained to fall between \pm 3 standard deviation $\sigma_{i}$. To place a shape $\mathbf{s}$ in the 3D space, an additional global transform is needed. We use the vector $\mathbf{g}=\left[t_{x}, t_{y}, t_{z}, \theta_{y}, \theta_{p}, \theta_{r}\right]$ to encode translation and rotation.

The vertices of the shape model are arranged in a triangulated mesh. Projecting the average shape $\mathbf{s}_{0}$ on an image of predefined size produces the template patch where the linear texture model lives. Such "shape-free" image is invariant to global motion and local facial deformations, i.e., each pixel always corresponds to the same semantic location on the face. The area $\mathbf{y}$ of an input image falling under the current shape model's instance is warped onto the shape-free patch through a piece-wise affine warp $W(\mathbf{y} ; \mathbf{g}, \mathbf{b})$, with a separate affine mapping for each triangle in the mesh, as described in [8]. Example shape-free images, with the triangulated mesh overlaid, are shown in Figure 2.

The warping can be applied to depth images with just minor expedients. The main problem of depth images is the fact that they often contain holes where the reconstruction was not successful and thus the depth value is undefined. To perform the bi-linear interpolation needed to warp a depth image onto the normalized patch, we only take into account pixels for which the depth information is available.

Denoting with $\mathbf{x}$ the set of pixels which fall on the shapefree patch, the linear texture model (we use the words texture and appearance interchangeably) is defined as:

$$
A(\mathbf{x})=A_{0}(\mathbf{x})+\sum_{i=1}^{m} \lambda_{i} A_{i}(\mathbf{x})
$$


with $A_{0}(\mathbf{x})$ being the average texture, $\lambda_{i}$ the appearance parameters, and $A_{i}(\mathbf{x})$ the eigenfaces, i.e., the principal components of the shape-free versions of the training images. We build the texture model in (2) only for the intensity channel and use the depth only for the regression, as will be explained in Section III-A. In fact, it is not trivial to perform PCA on a set of images with missing values, though methods exist to circumvent this issue [32].

Usually, AAMs are fit to new data by minimizing the image difference between the texture model reconstruction and the shape-free version of the input image, given the current shape. Recently, the work of [5] investigated new ways of performing the fitting to a new image. The idea is to use standard regression methods to directly learn a mapping between the shape-free version of the input image and additive updates to the model's parameters, usually resulting in a more efficient fitting. The authors investigated the use of both Boosting and Random Regression Forests (RRFs), with the latter proving less prone to over-fitting.

In this paper, we focus on estimating the shape model's parameters, i.e., $\mathbf{g}$ and $\mathbf{b}$, given a new scan of a unseen face, using random forests guided by both the intensity and the depth channel. To the best of our knowledge, there is no work in the literature which leverages depth data within an AAM framework. The texture model in the AAM is used only when the shape optimization is completed: After alignment, the resulting shape-free image is projected onto the texture PCA space and the corresponding appearance parameters found. The sum of squared pixel differences between the shape-free version of the test image and the reconstructed model is used as a measure of the goodness of the fit.

\section{A. Random Forests for 3D AAM Fitting}

In order to train our random forest-based regressors, we start with a set of facial scans, containing both the depth and intensity channels. The faces are annotated with the global motion parameters $\mathbf{g}_{g t}$, and the 3D positions of all landmarks, from which we extract the ground truth shape parameters $\mathbf{b}_{g t}$.

We build Independent Sequential AAMs with each regressor separately trained on facial scans where the optimal model parameters are randomly displaced within a predefined range [5], [33]. For the global parameters the range of displacements is chosen depending to the expected quality of the initialization procedure used at test time. For example, the head pose estimation method described in [28] returns errors below $10 \mathrm{~mm}$ for the nose localization and below 10 degrees for the rotation angles, when applied to high resolution scans. The PCA parameters' perturbations from the true values are randomly and uniformly drawn between \pm 0.5 standard deviation.

In their experiments on 2D images, the authors of [5] report best results when using a separate forest for each parameter. Our goal is real time processing, for example as initialization for tracking algorithms like [2]. We therefore divide the parameters to be optimized between 2 forests only: One for the global transform (translation and rotation) and one for the PCA shape model (40 parameters).
For a specific forest, we extract a number of training samples from an annotated scan by deforming its corresponding model's parameters and recording the resulting 3D shape and both depth and intensity shape-free images. A training sample therefore contains the pair of normalized images (depth+intensity), the deformed shape $\mathbf{s}_{d e f}$, and the set of real-valued offsets to the ground truth model parameters $\mathbf{d}$. In symbols, we build a tree starting from a set of samples $\left\{\mathscr{S}=\left(\mathscr{I}, \mathbf{s}_{\text {def }}, \mathbf{d}\right)\right\}$, where $\mathscr{I}$ contains both the depth and the intensity shape-free images.

The process of building a tree follows the standard random forest framework [7]. That is, starting from the root, every non-leaf node in the tree is assigned a binary test chosen from a large set of randomly generated tests $\{\phi\}$. The selected tests split the training data trying to reduce the variance of the clusters of samples sent to the children nodes. We stop growing a tree when either the maximum depth is reached (20), or less than a certain number of training samples are left (20). In both cases, we then create a leaf $L$, where we store the multivariate Gaussian distribution computed over the remaining training samples' offsets.

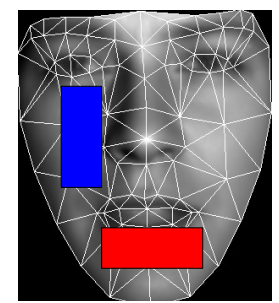

(a)

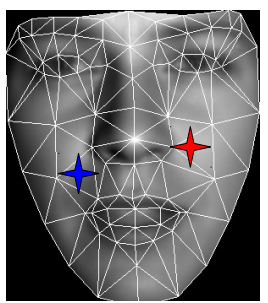

(b)
Fig. 2. Tests used at the nodes of the binary trees. (a) Difference between the average value of two rectangular region. (b) Pixel difference, within the template's triangles.

While the authors of [5] only investigate standard Haarlike features, we extend the possible kinds of tests available for optimization at each non-leaf node. A first kind of test is the difference between the average pixel values of two rectangles $F_{1}$ and $F_{2}$ defined within the shape-free patch, compared against a threshold $\tau$. We define such tests as $\phi_{c h, F_{1}, F_{2}, \tau}^{1}(\mathscr{I})$ :

$$
\left|F_{1}\right|^{-1} \sum_{q \in F_{1}} \mathscr{I}^{c h}(q)-\left|F_{2}\right|^{-1} \sum_{q \in F_{2}} \mathscr{I}^{c h}(q)>\tau,
$$

where $c h \in\{0,1\}$ indicates the channel (intensity or depth). A sample satisfying the test goes to the right, otherwise left child. Tests like these, exemplified by Figure 2(a), are in fact a generalization of Haar-like features and have been previously used for face analysis [19], [28].

A second type of test involves pixel differences, as in the work of [24], among others. The pixels' locations though are not just randomly sampled from the shape-free image patch, but determined by a pair of triangles $t_{1}$ and $t_{2}$, and by the barycentric coordinates within them. This has the advantage of selecting more often locations within the smaller triangles which surround salient features like the 
mouth or the eyes. Less useful areas of the face, such as the cheeks, are covered by larger triangles and thus will see a lower number of tests within their areas. We define these tests as $\phi_{c h, t_{1}, t_{2}, \alpha_{1}, \alpha_{2}, \beta_{1}, \beta_{2}, \tau}^{2}(\mathscr{I})$

$$
\mathscr{I}^{c h}(p)-\mathscr{I}^{c h}(q)>\tau \text {. }
$$

In the above equation, $p=\alpha_{1} t_{1}^{a}+\beta_{1} t_{1}^{b}+\left(1-\alpha_{1}-\beta_{1}\right) t_{1}^{c}$ and $q=\alpha_{2} t_{2}^{a}+\beta_{2} t_{2}^{b}+\left(1-\alpha_{2}-\beta_{2}\right) t_{2}^{c}$, while $t_{i}^{a}, t_{i}^{b}, t_{i}^{c}$ are the i-th triangles' vertices, and $\alpha_{i}, \beta_{i}$ are the corresponding barycentric coordinates. The channel $c h$ again indicates whether the intensity or the depth shape-free image is used. Figure 2(b) shows one such test.

A third and last kind of test compares the $\mathrm{z}$ value at a vertex $i$ of the shape model instance $s$ and the corresponding depth value at such vertex's $2 \mathrm{D}$ location $W\left(z_{i} ; \mathbf{g}, \mathbf{b}\right)$ on the shape-free depth image. Such tests are only defined on the depth channel and can be expressed as $\phi_{i, \tau}{ }^{3}(\mathscr{I})$ :

$$
z_{i}-\mathscr{I}^{1}\left(W\left(z_{i} ; \mathbf{g}, \mathbf{b}\right)\right)>\tau
$$

During training, the test for a node is chosen as the best of a large number of binary tests, generated by randomly sampling the type, the features channel, etc. The goodness of a test is quantified by a measure computed over the labels of the patches directed to the left and right child. That is, we select a test out of the generated set $\{\phi\}$ such that:

$$
\phi^{*}=\operatorname{argmin}\left(\mathscr{U}\left(S_{l}\right)+\mathscr{U}\left(S_{r}\right)\right) .
$$

In the above, $S_{l}$ and $S_{r}$ are the sets of patches which the chosen test directs towards the left, respectively right child node. The measure $\mathscr{U}(S)$ is defined based on the entropy of the continuous patch labels. Assuming the real-valued offsets d to be realizations of $D$-variate Gaussians, we can represent the labels in a set $S$ as:

$$
p(\mathbf{d})=\mathscr{N}(\mathbf{d} ; \overline{\mathbf{d}}, \Sigma)
$$

where $\mathbf{d}$ can refer to both global and local shape parameters' updates. The differential entropy $H(S)$ for a $D$-variate Gaussian distribution being

$$
H(S)=\frac{1}{2} \log \left((2 \pi e)^{D}|\Sigma|\right)
$$

we define our measure as:

$$
\mathscr{U}(S)=|S| \cdot \log (|\Sigma|) \propto|S| \cdot H(S) .
$$

Minimizing the sum of Equation (9) computed over the two sets $S_{l}$ and $S_{r}$ favors splits with more peaked (i.e., with lower covariance) Gaussian distributions of labels d. For the global parameters $\mathbf{g}$, some kind of correlation between them could be allowed (as done in [28]), and the determinant of the covariance matrices minimized. However, the PCA parameters are by construction uncorrelated and, in practice, we minimize the trace of the covariance matrices $\Sigma$ both for the global and local parameters' offsets.

When a leaf is created, it stores the Gaussian distribution $p(\mathbf{d})=\mathscr{N}(\mathbf{d} ; \overline{\mathbf{d}}, \Sigma)$ computed over the remaining labels.
At test time, we assume the shape model to be initialized close to the optimal position. Then, given the current instance of the model's parameters, both depth and intensity images are warped onto the shape-free patch and sent down all trees in each forest. The forests are visited in a sequence, starting from the one optimizing global parameters and continuing with the forest performing the regression over the PCA parameters controlling local deformations. The shapefree images are evaluated by the tests stored at the nodes and guided towards the left or right child until a leaf is reached. There, the corresponding Gaussians are retrieved and averaged over all trees in the forest, producing the updates to be added to the current (for iteration it) model's parameters:

$$
\begin{array}{r}
\mathbf{g}_{i t+1}=\mathbf{g}_{i t}+\overline{\mathbf{d}_{g}} \\
\mathbf{b}_{i t+1}=\mathbf{b}_{i t}+\overline{\mathbf{d}_{b}},
\end{array}
$$

where $\mathbf{d}_{g}$ and $\mathbf{d}_{b}$ represent the global, respectively local parameters' updates. The process iterates until either the change is smaller than a threshold or a maximum number of iterations is reached.

At the end of the fitting process, the shape-free version of the texture image is projected onto the PCA space and the appearance reconstructed. The error image between this reconstruction and the shape-free version of the input image represents a measure of the goodness of the fit and divergence can be automatically detected from it.

\section{RESULTS AND EXPERIMENTS}

In order to assess the quality of the proposed approach to real time, frame-independent face alignment, we ran experiments targeted at evaluating the robustness of the fitting to unseen 3D scans of faces displaying facial expressions.

Unless otherwise specified, we initialize the mean shape $(\mathbf{b}=0)$ at a fixed distance from the ground truth location along a random direction, and with the rotation parameters set to zero. As fitting error, we use the Euclidean distance between each model's point and the ground truth.

Random forests show their power when trained on large databases. We therefore needed many depth+intensity images of several people, with different expressions, annotated with the facial feature points we are interested in.

We use the $B 3 D(A C)^{2}$ database [34], where 14 people utter a set of 40 sentences in front of a 3D scanner, both in neutral and (elicited) emotional tone. Each frame (out of over 100K) comes annotated with a dense, generic face model, deformed to fit both identity and expression. By selecting a set of 88 landmarks on the generic template, we automatically obtained their 3D locations for the whole database.

In addition, we also use the database of [35] (BU3DFE), containing 100 subjects each captured while posing the six basic expressions of emotions [36], with varying levels of intensity. This second database has a greater number of identities and the represented facial dynamics are also larger, though less naturalistic. The $B U 3 D F E$ data comes in the form of textured $3 \mathrm{D}$ models, annotated with the locations 

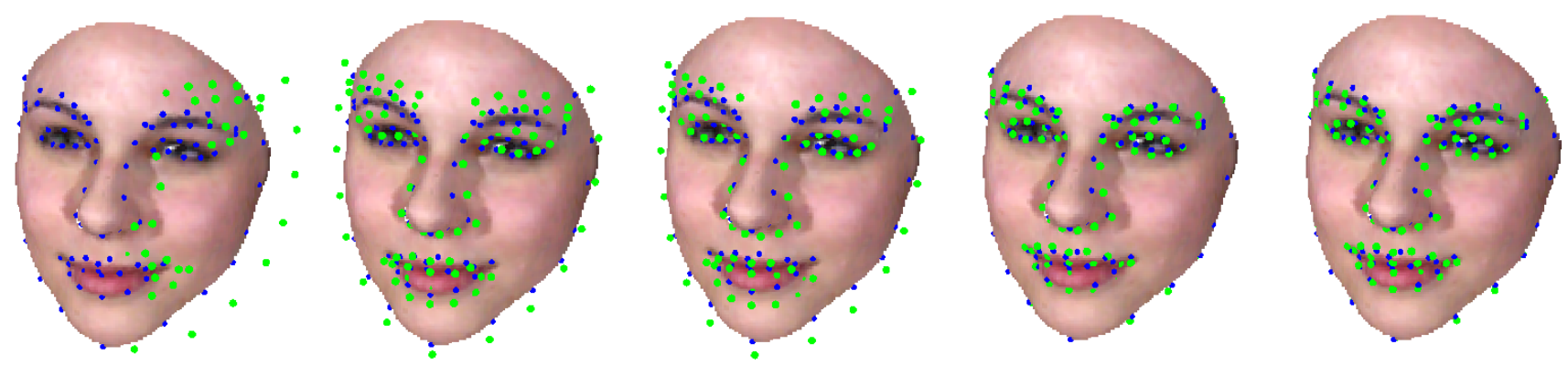

Fig. 3. Fitting procedure. From left to right, the green spheres representing the current model's instance move from an initially perturbed position to a configuration close to the ground truth locations (in blue).

of 83 facial features. We generated our training and testing sets by rendering the textured models undergoing rotations uniformly sampled within -20 and 20 degrees yaw, \pm 10 pitch, and \pm 5 roll. These intervals do not represent hard limits for the algorithm and larger rotations could anyway be handled using view-based models, selected based on the estimated head pose. We also randomly displaced the model along the $\mathrm{Z}$ axis, centering it between 60 and $80 \mathrm{~cm}$ from the camera. Each scan was rendered 10 times, resulting in a dataset of $25 \mathrm{~K}$ depth+intensity, $640 \times 480$ images, with the faces being around 130x150 pixels on average.

On both datasets, we performed a 5-fold, subjectindependent cross-validation, i.e., the subjects used for training are never found in the test set. For each experiment, we constructed the AAM using 1000 randomly selected images, capping the number of PCA modes to 40 . We trained two independent forests: One for the global motion parameters, containing 20 trees, and one for the local PCA deformations, with 30 trees. Each tree was built from 1000 randomly sampled images, each distorted 10 times.

Figure 3 shows the iterations of the algorithm when applied to a test face from the $B U 3 D F E$ database. The green spheres represent the model's points: From left to right, they are guided from a displaced initialization towards the ground truth locations, marked in blue.

The plot in Figure 4 shows the average error (Euclidean distance computed over all test samples in all folds and over all feature points) as a function of the introduced deformations to the translation vector at initialization. The different curves show how the performance of the system changes depending on the feature channels used, and for the two separate databases. We notice that adding depth substantially reduces the errors, especially when the initialization of the shape model is far from the ground truth translation. In fact, the intensity channel does not seem to add much to the performance of the system, suggesting that it would work also under suboptimal lighting conditions. The interested reader can find a comparison between the original AAM algorithm and an implementation based on regression forest in [5].

Figure 6 shows some examples of successful fits on expressive frames from the $B U 3 D F E$ database. In green are the estimated model vertices, while in blue the ground

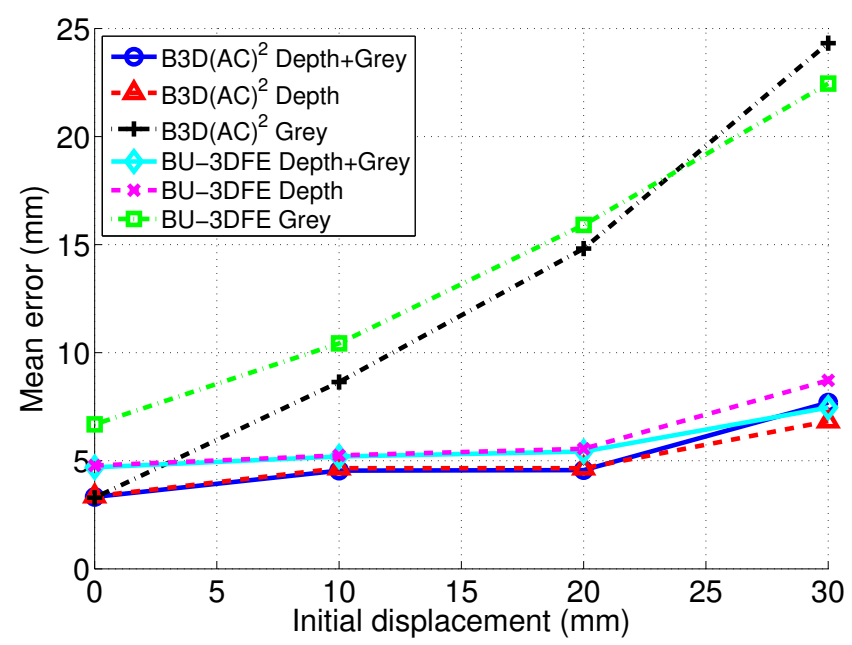

Fig. 4. Mean error (computed over all the 83 model points) as a function of the initial maximum offset from the ground truth face location.

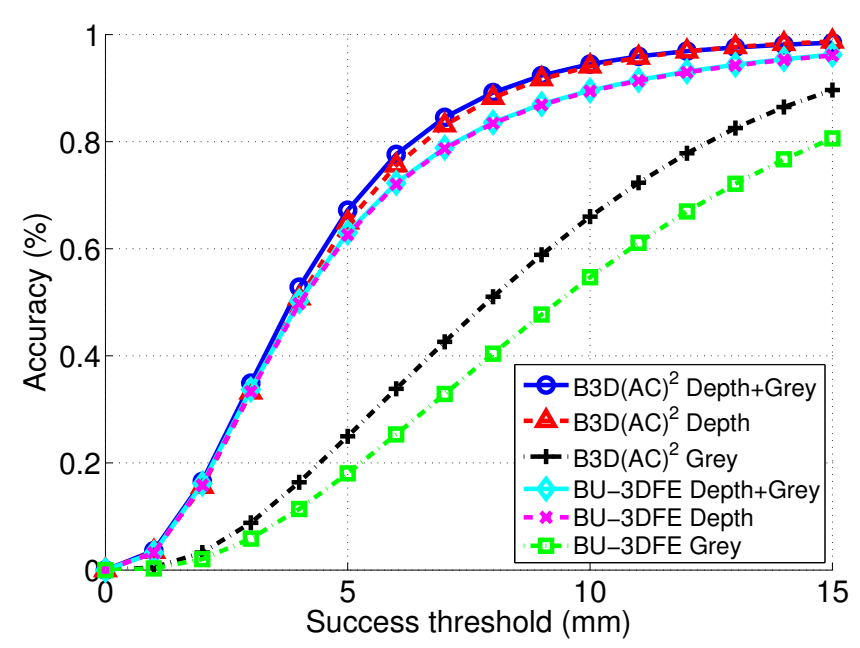

Fig. 5. Percentage of correctly aligned faces as function the error threshold in millimeters.

truth. For what concerns the $B 3 D(A C)^{2}$ database, Figure 7 gives some example frames where the subjects are speaking emotionally; note how the automatically generated ground truth is not perfect, especially around the eyebrows. 

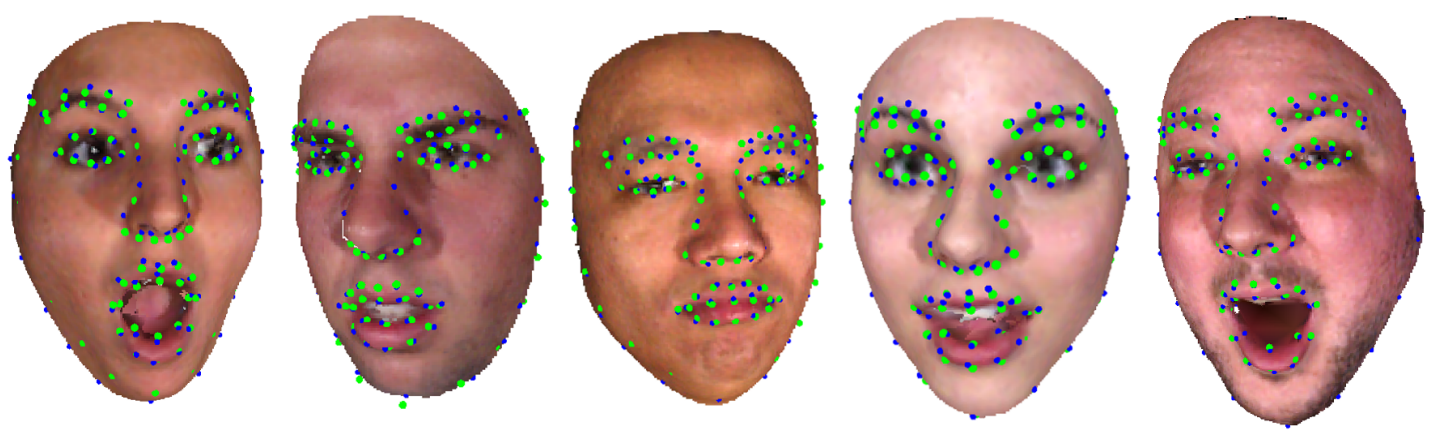

Fig. 6. Examples of successfully aligned faces from the BU3DFE database. The estimated points are in green, while the ground truth is in blue.
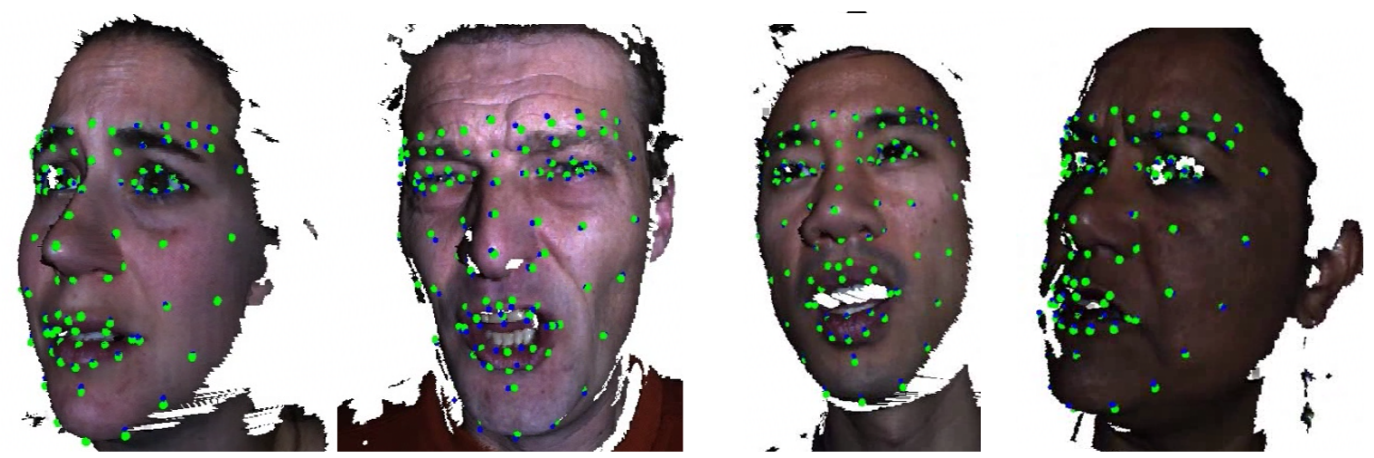

Fig. 7. Frames from the $B 3 D(A C)^{2}$ database. It can be noted how the (automatically generated) ground truth is not perfect, e.g., for the eyebrows.

\begin{tabular}{|l|c|c|}
\hline fiducial & success \% $(5 / 10 \mathrm{~mm})$ & mean \pm std error $(\mathrm{mm})$ \\
\hline innEyeL & $86.0 / 98.1$ & $3.4 \pm 2.1$ \\
outEyeL & $65.7 / 99.0$ & $4.4 \pm 1.9$ \\
innEyeR & $77.9 / 99.4$ & $3.8 \pm 1.9$ \\
outEyeR & $54.9 / 98.3$ & $4.8 \pm 2.3$ \\
noseL & $83.8 / 99.9$ & $3.3 \pm 1.7$ \\
noseR & $90.1 / 100.0$ & $2.9 \pm 1.7$ \\
mouthL & $54.5 / 90.7$ & $5.3 \pm 3.1$ \\
upLip & $82.1 / 99.8$ & $3.5 \pm 1.7$ \\
mouthR & $50.3 / 92.8$ & $5.3 \pm 2.9$ \\
lowLip & $61.7 / 82.1$ & $6.2 \pm 5.8$ \\
outBrowL & $27.5 / 82.0$ & $7.1 \pm 3.1$ \\
innBrowL & $79.0 / 99.8$ & $3.3 \pm 1.8$ \\
innBrowR & $87.7 / 100.0$ & $3.1 \pm 1.5$ \\
outBrowR & $34.7 / 71.5$ & $7.5 \pm 4.3$ \\
\hline
\end{tabular}

TABLE I

RESULTS OF 5-FOLD, SUBJECT-INDEPENDENT CROSS-VALIDATION ON THE $B 3 D(A C)^{2}$ DATABASE, WITH THE SHAPE MODEL INITIALIZED $10 \mathrm{MM}$ AWAY FROM THE GROUND TRUTH POSITION.

Tables I and II report the performance of the system (when both intensity and depth are used) for a subset of model vertices corresponding to important landmarks, i.e., the 14 fiducials chosen for the 3D facial feature detector proposed by [28]. In particular, Table I shows success rate plus mean and standard deviation of the Euclidean error for each fiducial, on the $B 3 D(A C)^{2}$, while Table II shows the corresponding results for the $B U 3 D F E$ faces. The landmarks which present the largest errors are the outer ends of the eyebrows. Our results are not far from those in [28], whose

\begin{tabular}{|l|c|c|}
\hline fiducial & success \% $(5 / 10 \mathrm{~mm})$ & mean \pm std error $(\mathrm{mm})$ \\
\hline innEyeL & $95.5 / 99.9$ & $2.6 \pm 1.8$ \\
outEyeL & $78.6 / 99.2$ & $3.6 \pm 2.4$ \\
innEyeR & $92.7 / 99.8$ & $2.8 \pm 2.0$ \\
outEyeR & $72.6 / 98.4$ & $4.0 \pm 2.8$ \\
noseL & $76.3 / 98.8$ & $3.9 \pm 2.0$ \\
noseR & $73.6 / 98.7$ & $4.1 \pm 2.2$ \\
mouthL & $67.3 / 92.6$ & $4.7 \pm 3.5$ \\
upLip & $83.1 / 97.2$ & $3.5 \pm 2.5$ \\
mouthR & $63.2 / 92.2$ & $4.9 \pm 3.6$ \\
lowLip & $66.8 / 92.4$ & $5.2 \pm 5.2$ \\
outBrowL & $49.7 / 89.4$ & $5.8 \pm 3.8$ \\
innBrowL & $76.8 / 98.5$ & $3.8 \pm 2.7$ \\
innBrowR & $73.2 / 98.1$ & $4.0 \pm 3.0$ \\
outBrowR & $45.7 / 86.0$ & $6.2 \pm 4.3$ \\
\hline
\end{tabular}

TABLE II

5-FOLD CROSS-VALIDATION RESULTS ON THE BU3DFE DATABASE.

processing time scales linearly with the number of fiducials, while the proposed approach can detect many more landmarks without affecting runtime. It must be noted that the algorithm of [28] only uses the depth channel; however, as noticeable from Figures 4 and 5, adding the intensity channel does not increase the performance of our approach.

As an additional experiment, we synthetically removed patches of depth and intensity images, to control the behavior of the system when some of the reconstruction is missing. Figure 8 shows some examples images where the estimation was correct despite the occlusions. The plot in Figure 9 gives an idea of how the error increases as the amount of 

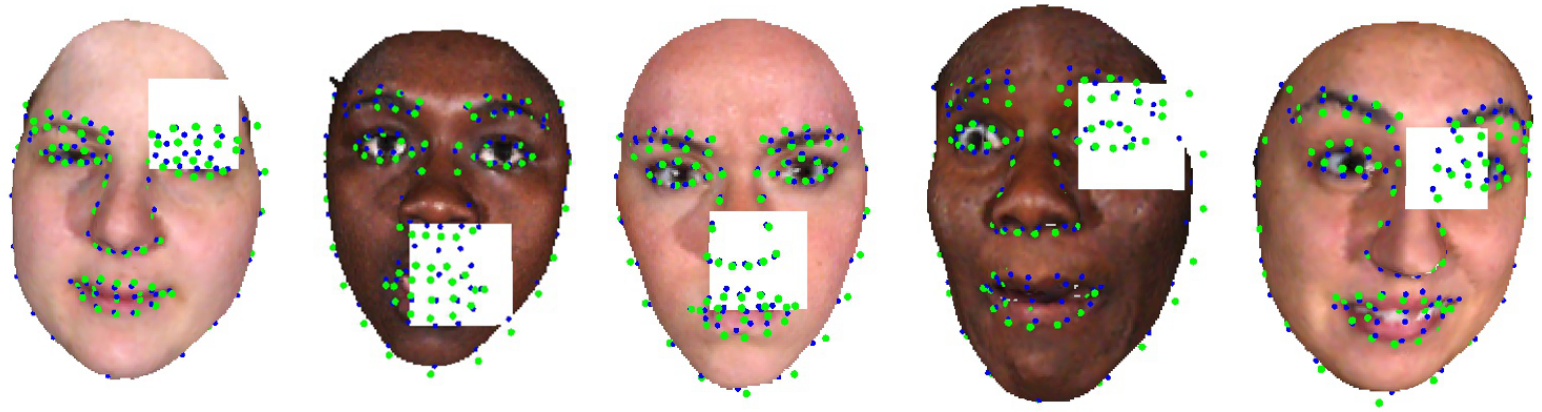

Fig. 8. Example images from $B U 3 D F E$ the where synthetically removed data did not influence the detection.

missing data (expressed in percentage of the area enclosed by the ground truth landmarks' positions) becomes greater. The two curves represent models initialized on the ground truth translation (blue, continuous), or $10 \mathrm{~mm}$ away from it (red, dashed). Some amount of missing data can be tolerated, i.e., when $15 \%$ of the face area is set to zero, the average error is still below 10 millimeters. Finally, Figure 10 shows some example cases from the $B U 3 D F E$ in which the algorithm converged to a wrong shape.

We tested our algorithm on a $2.6 \mathrm{GHz}$ machine, without particular optimization efforts, setting the maximum number of iterations to 15 for the global forest and 20 for the local one. Our AAMs are built such that the template patch has a width of 80 pixels. In these settings, processing one face scan takes on average $39 \mathrm{~ms}$, i.e., about $25 \mathrm{fps}$.

We directly applied the described system to range scans coming from the 3D scanner of [1]. Each frame is processed independently and initialization is achieved using the head pose estimation algorithm described in [28]. Some frames extracted from the accompanying video are shown in Figure 11, with subjects not present in the training set touching their face and displaying facial expressions.

\section{Conclusions}

We presented a system for robust real time face alignment. We use the standard AAM framework and employ random forests to learn the regression between deformed training instances and the necessary updates to the parameters, as originally proposed in [5].

Our contributions include the real time implementation and the use of both intensity and depth images, the latter becoming ever more available. We also propose new features to be employed as binary tests within the trees in the forest.

We evaluated our approach on publicly available 3D databases, in a subject-independent cross-validation. Our experiments show that using depth greatly increases performance. The low errors achieved and the fast computation are valuable steps forward for achieving robust, drift-free tracking of facial features, a vital step for many useful applications ranging from recognition to animation.

Future work would see the extension of the proposed system to handle noisy depth data coming from consumerlevel cameras such as Microsoft Kinect or Asus Xtion.

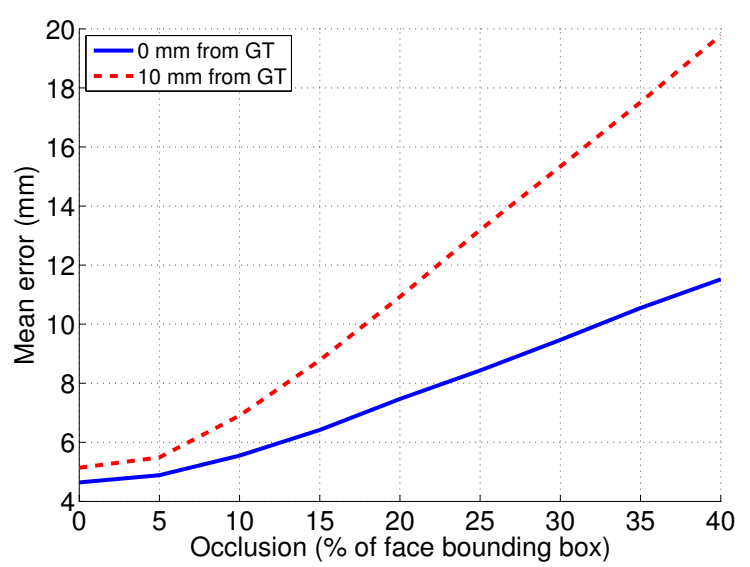

Fig. 9. Mean Euclidean error as a function of the amount of synthetically removed data from the depth and intensity images.
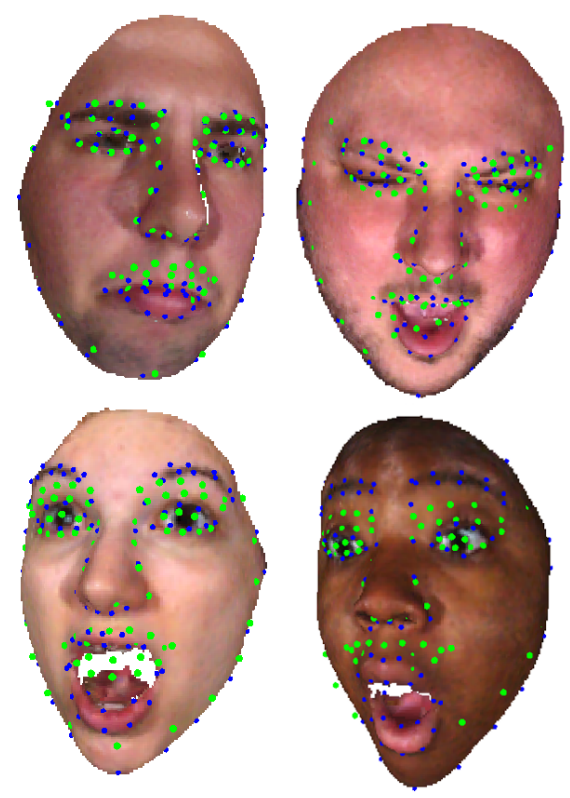

Fig. 10. Examples of wrongly aligned model on expressive faces from the $B U 3 D F E$ database. 

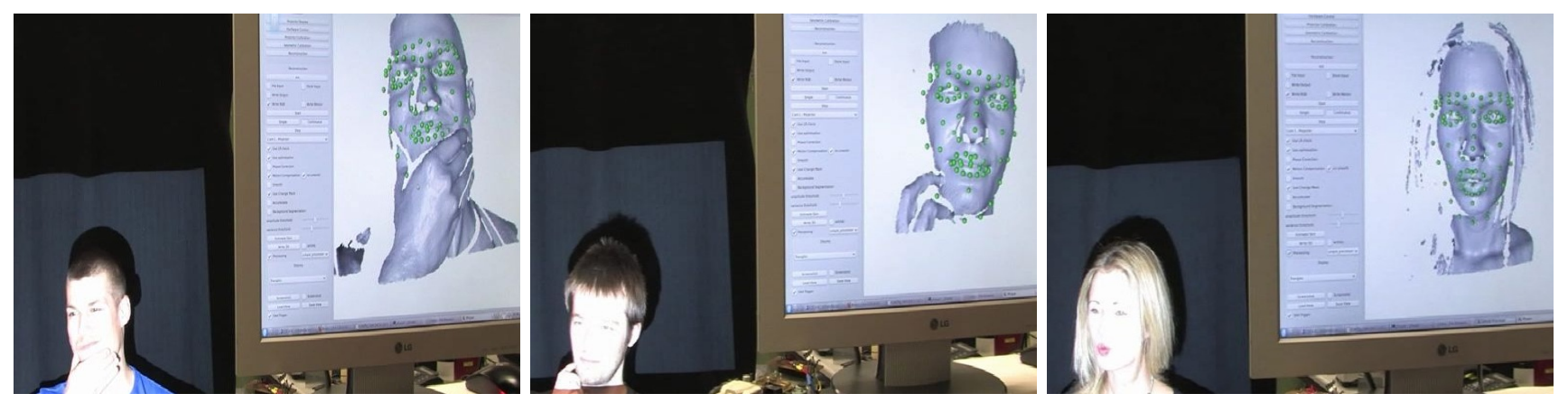

Fig. 11. Real time results of the system applied directly on the output of the 3D scanner of [1].

\section{REFERENCES}

[1] Weise, T., Leibe, B., Van Gool, L.: Fast 3d scanning with automatic motion compensation. In: Computer Vision and Pattern Recognition. (2007)

[2] Weise, T., Li, H., Van Gool, L., , Pauly, M.: Face/off: Live facial puppetry. In: Symposium on Computer Animation. (2009)

[3] Weise, T., Bouaziz, S., Li, H., Pauly, M.: Realtime performance-based facial animation. ACM Trans. Graph. 30 (2011) 77:1-77:10

[4] Breidt, M., Buelthoff, H., Curio, C.: Robust semantic analysis by synthesis of $3 \mathrm{~d}$ facial motion. In: Automatic Face and Gesture Recognition. (2011)

[5] Sauer, P., Cootes, T., Taylor, C.: Accurate regression procedures for active appearance models. In: British Machine Vision Conference. (2011)

[6] Cootes, T.F., Edwards, G.J., Taylor, C.J.: Active appearance models. IEEE Transactions on Pattern Analysis and Machine Intelligence 23 (2001) 681-685

[7] Breiman, L.: Random forests. Machine Learning 45 (2001) 5-32

[8] Matthews, I., Baker, S.: Active appearance models revisited. International Journal of Computer Vision 60 (2003) 135-164

[9] Cootes, T.F., Wheeler, G.V., Walker, K.N., Taylor, C.J.: View-based active appearance models. Image and Vision Computing 20 (2002) $657-664$

[10] Blanz, V., Vetter, T.: A morphable model for the synthesis of $3 \mathrm{~d}$ faces. In: SIGGRAPH. (1999) 187-194

[11] Gross, R., Matthews, I., Baker, S.: Generic vs. person specific active appearance models. Image and Vision Computing 23 (2005) 1080 2093

[12] Valstar, M., Martinez, B., Binefa, X., Pantic, M.: Facial point detection using boosted regression and graph models. In: Computer Vision and Pattern Recognition. (2010)

[13] Amberg, B., Vetter, T.: Optimal landmark detection using shape models and branch and bound slides. In: International Conference on Computer Vision. (2011)

[14] Belhumeur, P.N., Jacobs, D.W., Kriegman, D.J., Kumar, N.: Localizing parts of faces using a consensus of exemplars. In: Computer Vision and Pattern Recognition. (2011)

[15] Cootes, T., Taylor, C., Cooper, D., Graham, J., et al.: Active shape models-their training and application. Computer vision and image understanding 61 (1995) 38-59

[16] Cristinacce, D., Cootes, T.: Automatic feature localisation with constrained local models. Journal of Pattern Recognition 41 (2008) 3054-3067

[17] Saragih, J.M., Lucey, S., Cohn, J.F.: Deformable model fitting by regularized landmark mean-shift. International Journal of Computer Vision 91 (2011) 200-215

[18] Baltrušaitis, T., Robinson, P., Morency, L.P.: 3d constrained local model for rigid and non-rigid facial tracking. In: Computer Vision and Pattern Recognition. (2012)

[19] Dantone, M., Gall, J., Fanelli, G., Van Gool, L.: Real-time facial feature detection using conditional regression forests. In: Computer Vision and Pattern Recognition. (2012)

[20] Zhao, X., Dellandréa, E., Chen, L., Kakadiaris, I.: Accurate landmarking of three-dimensional facial data in the presence of facial expressions and occlusions using a three-dimensional statistical facial feature model. IEEE transactions on systems, man, and cybernetics (2011)
[21] Nair, P., Cavallaro, A.: 3-d face detection, landmark localization, and registration using a point distribution model. IEEE Transactions on multimedia 11 (2009) 611-623

[22] Chang, K.I., Bowyer, K.W., Flynn, P.J.: Multiple nose region matching for $3 \mathrm{~d}$ face recognition under varying facial expression. IEEE Transactions on Pattern Analysis and Machine Intelligence 28 (2006) 1695-1700

[23] Creusot, C., Pears, N., Austin, J.: Automatic keypoint detection on 3d faces using a dictionary of local shapes. In: 3DIMPVT. (2011)

[24] Gall, J., Yao, A., Razavi, N., Gool, L.V., Lempitsky, V.: Hough forests for object detection, tracking, and action recognition. IEEE Transactions on Pattern Analysis and Machine Intelligence 33 (2011) 2188-2202

[25] Shotton, J., Fitzgibbon, A., Cook, M., Sharp, T., Finocchio, M., Moore, R., Kipman, A., Blake, A.: Real-time human pose recognition in parts from single depth images. In: Computer Vision and Pattern Recognition. (2011)

[26] Girshick, R., Shotton, J., Kohli, P., Criminisi, A., Fitzgibbon, A.: Efficient regression of general-activity human poses from depth images. International Conference on Computer Vision (2011)

[27] Yao, A., Gall, J., Fanelli, G., Van Gool., L.: Does human action recognition benefit from pose estimation? In: British Machine Vision Conference. (2011)

[28] Fanelli, G., Dantone, M., Gall, J., Fossati, A., Van Gool, L.: Random forests for real time $3 \mathrm{~d}$ face analysis. International Journal of Computer Vision (2012)

[29] Fanelli, G., A.Yao, Noel, P.L., Gall, J., Van Gool, L.: Hough forest-based facial expression recognition from video sequences. In: International Workshop on Sign, Gesture and Activity. (2010)

[30] Saragih, J., Goecke, R.: Learning active appearance models from image sequences. In: HCSNet workshop on Use of vision in humancomputer interaction. (2006)

[31] Breitenstein, M.D., Kuettel, D., Weise, T., Van Gool, L., Pfister, H.: Real-time face pose estimation from single range images. In: Computer Vision and Pattern Recognition. (2008)

[32] De la Torre, F., Black, M.J.: Robust principal component analysis for computer vision. In: International Conference on Computer Vision. (2001)

[33] Saragih, J., Goecke, R.: Iterative error bound minimisation for aam alignment. In: International Conference on Pattern Recognition. (2006)

[34] Fanelli, G., Gall, J., Romsdorfer, H., Weise, T., Van Gool, L.: A 3-d audio-visual corpus of affective communication. IEEE Transactions on Multimedia 12 (2010) 591 - 598

[35] Yin, L., Wei, X., Sun, Y., Wang, J., Rosato, M.J.: A 3d facial expression database for facial behavior research. In: Automatic Face and Gesture Recognition. (2006)

[36] Ekman, P.: Constants across cultures in the face and emotion. Journal of personality and social psychology 17 (1971) 124-129 